\title{
LA ALTA VELOCIDAD FERROVIARIA EN LA UNIÓN EUROPEA. SU IMPACTO URBANO EN FRANCIA Y ESPAÑA
}

\author{
Víctor ESTEBAN MARTÍN \\ Departamento de Geografía y Ordenación del Territorio \\ Universidad de Zaragoza
}

\begin{abstract}
Resumen: En la actualidad, existen en la Unión Europea distintos sistemas de alta velocidad ferroviaria. La mayor densidad de población y el mayor equilibrio de sus redes urbanas, han permitido a Italia y Alemania dotarse de servicios de alta velocidad que, al contrario de lo que sucede en Francia y España, se inscriben de manera armoniosa dentro del conjunto de la red ferroviaria.

Por otra parte, después de más de quince años de la puesta en marcha de la primera línea de alta velocidad en Europa, ya pueden empezar a extraerse las primeras conclusiones en lo referente al impacto socioeconómico que tiene este modo de transporte en el medio urbano. Si bien las ciudades servidas por el T.A.V. (Tren de Alta Velocidad), experimentan una mejora general de su accesibilidad, estas ven al mismo tiempo degradarse sus servicios de tren convencional y avión en aquellas líneas donde el T.A.V. ejerce su competencia. La llegada de la alta velocidad, suele ser aprovechada para remodelar urbanísticamente el barrio de la estación, zona en general, muy degradada. Por otra parte, la presencia del T.A.V. no suele ser considerada por las empresas de terciario superior como la principal causa para localizarse en el barrio de la estación, si bien es un factor muy tenido en cuenta por aquellas empresas que guardan relación con otros núcleos urbanos que se encuentran en la línea de alta velocidad. Por lo que respecta al turismo, la posibilidad de hacer el viaje de ida y vuelta en el mismo día, afecta negativamente al número de pernoctas registradas en la ciudad. El turismo de negocios y de congresos se ve, en cambio muy beneficiado.
\end{abstract}

Palabras clave: Accesibilidad, barrio de la estación, ciudad, España, Francia, localización empresarial, Tren de Alta Velocidad, turismo, Unión Europea.

\begin{abstract}
Nowadays, different High Speed Train systems exists in the European Union. Italy and Germany have made good use of their high population densities and their balanced urban networks for having High Speed services that fit harmouniosely whithin the whole train network.

After more than 15 years of High Speed Trains in Europe, the first social and economic conclussions about the urban impact of this new way of transport can be seen. Cities served by T.G.V. show a general improvement in their accessibility. However, this may be to the detriment of traditional train and plane services in routes where they are in direct competition with the TGV. In general, councils make the most of the arrival of High Speed Trains for assisting the recovery of degraded areas like station quarters. The presence of the T.G.V. is not considered, by quaternary sector companies, as the most important reason to locate in the area surrounding the station. However, this is an important consideration for companies
\end{abstract}


wishing to conduct business with others located within urban centres along high speed rail links. Business and Congress Tourism may benefit from potentially greater accessibility. However, this may reduce the need for people to require overnight accomodation within a particular city due to the increased ability of completing a round trip during the course of a single day.

Key words: accessibility, city, company location, European Union, France, High Speed Train, Spain, tourism, station surroundings.

\section{INTRODUCCIÓN ${ }^{1}$}

Al igual que el resto de las infraestructuras de transporte, la alta velocidad ferroviaria es la consecuencia de un contexto social, económico y político que determina su forma y su impacto sobre el espacio. Está muy extendida la idea de que una nueva infraestructura de transporte otorga abundancia al mismo tiempo que asegura el desplazamiento de personas y bienes. Esta percepción fue reforzada por la distribución geográfica de las actividades industriales producida durante el siglo pasado, según la cual éstas se inscribían sobre los espacios provistos de infraestructuras portuarias o ferroviarias. Consumiendo y elaborando productos pesados, estas industrias no podían, en efecto, desarrollarse sin las infraestructuras de transporte (BONNAFOUS, 1981). Con este contexto, el concepto de efecto estructurante, aparecido entre los economistas espaciales franceses en 1964, establecía la existencia de una relación de causalidad entre la infraestructura y los fenómenos económicos y espaciales concomitantes. Sin embargo, los estudios llevados a cabo sobre las autopistas y después sobre las líneas de alta velocidad, ponen en entredicho la automaticidad de las transformaciones inducidas por las nuevas infraestructuras de transporte y niegan la existencia de una relación directa e inmutable entre la nueva infraestructura y la aparición de fenómenos económicos dados.

De la creencia simplista, según la cual la realización de una infraestructura de transporte aportaba riqueza y prosperidad, se ha pasado a la elaboración de $\mathbf{u}$ discurso donde se subraya la necesidad de acompañar a las nuevas infraestructuras de estrategias de valorización (MANNONE, 1995).

\footnotetext{
${ }^{1} \mathrm{El}$ presente artículo recoge las principales conclusiones de la tesis de licenciatura, Zaragoza y Nancy: dos ciudades frente a la perspectiva de la alta velocidad ferroviaria, codirigida por los profesores Etienne Auphan y Vicente Bielza de Ory, defendida a finales de septiembre de 1996 y premiada en 1998 con Premio Extraordinario de Licenciatura. Este trabajo fue posible gracias a una estancia en la Universidad de Nancy 2 durante el curso 1995-96, dentro del marco del programa Erasmus/Socrates. La ayuda del codirector de tesina, el profesor Etienne Auphan, uno de los principales estudiosos del impacto territorial de la alta velocidad, fue inextimable para la elaboración de este trabajo.
} 


\section{LA ALTA VELOCIDAD FERROVIARIA EN LA UNIÓN EUROPEA}

Los primeros trenes de alta velocidad entraron en servicio en Japón en 1964 y en Francia en el año 1981. A medida que éstos se han ido desarrollando, la alta velocidad se ha revelado para el transporte de viajeros como una alternativa al avión y al tren tradicional.

La velocidad que alcanzan estos nuevos trenes varía según las características geográficas, demográficas y económicas de las zonas a servir. Esta velocidad es generalmente superior a $160 \mathrm{~km} / \mathrm{h}$ y puede incluso pasar de los $300 \mathrm{~km} / \mathrm{h}$. El aumento de la velocidad, la posibilidad de servir el centro de las ciudades y la puesta en marcha de un nuevo material ferroviario han permitido dar salida a tráficos de intensidad elevada. Estas características permiten igualmente una mejora de la calidad del servicio, que se deja sentir en la reducción del tiempo de viaje, el confort, la seguridad y el acceso a diversos servicios. Sin embargo, esta mejora no siempre se experimenta por lo que concierne a las frecuencias.

En la actualidad, existen en Europa distintos sistemas de alta velocidad ferroviaria que otorgan soluciones diferentes por lo que respecta a la mayor o menor densidad de servicio en el territorio por parte del efecto alta velocidad.

\section{Los sistemas alemán e italiano}

Podemos afirmar que, en su actual forma, los sistemas italiano y alemán, permiten una integración considerable entre la red ferroviaria clásica y la red de alta velocidad, lo que potencia en su conjunto al transporte ferroviario. Lo anterior es resultado de una serie de factores que han permitido una concepción de la alta velocidad menos desterritorializadora que la francesa o la española. En efecto, tanto Alemania como Italia no poseen una capital macrocéfala y tienen sistemas de ciudades que pueden ser calificados como densos y equilibrados, lo que ha repercutido en la posibilidad de disponer de unas redes ferroviarias en forma de malla. Además, la fuerte densidad de población de estos países permite una difusión del tráfico ferroviario y una calidad del servicio relativamente altos.

De esta forma, al contrario de lo que sucede en Francia y en España, la causa de la puesta en servicio de la alta velocidad italiana y alemana no ha sido la concentración del tráfico sobre los ejes saturados sino la reorientación de los flujos de transporte en el caso alemán y la feroz competencia de la autopista en el caso italiano.

En estos países, el tren de alta velocidad (TAV) es complementado por los trenes clásicos rápidos ligeros así como por ciertos trenes de mercancías que utilizan total o parcialmente las nuevas líneas férreas. 


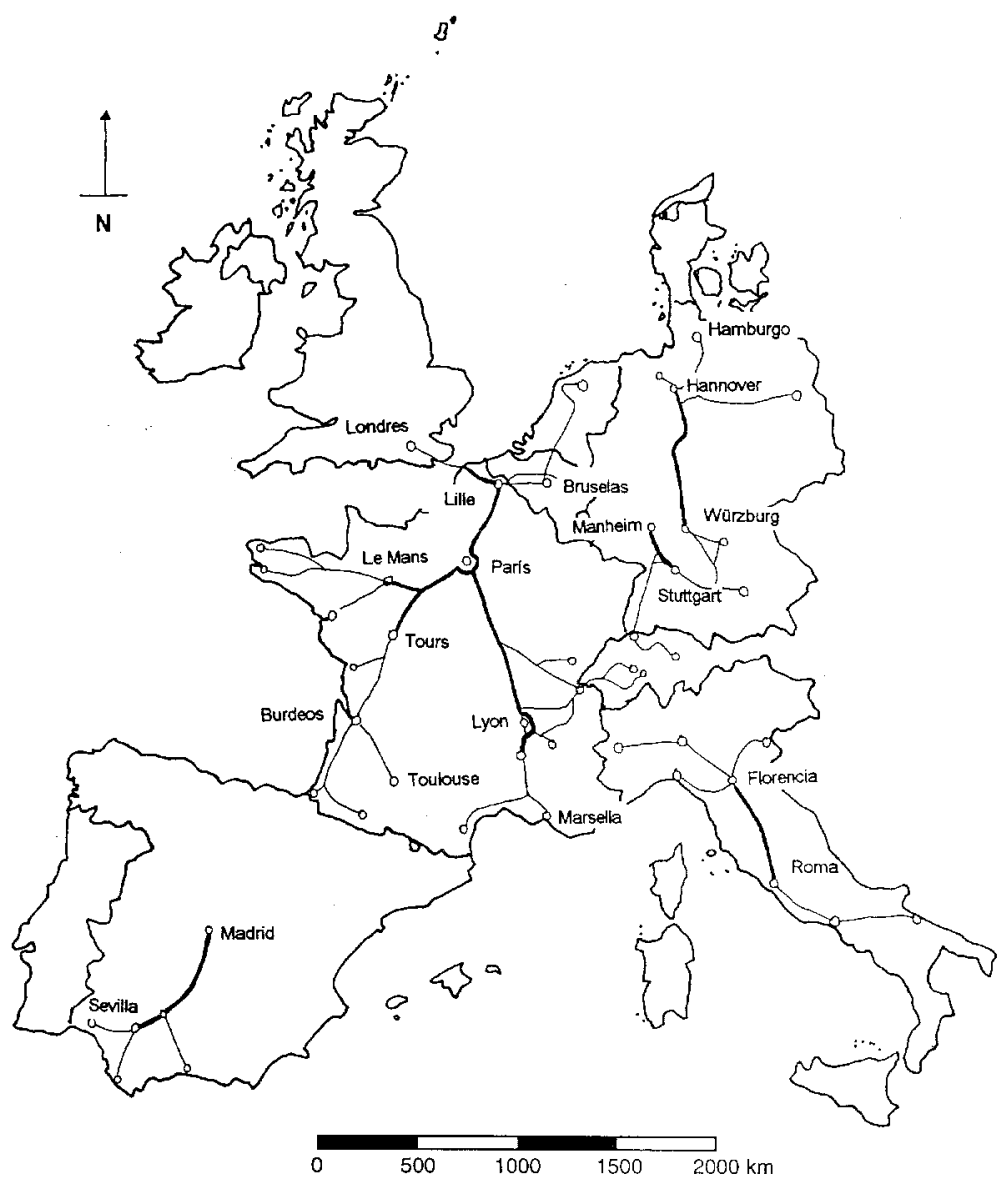

Figura 1.- La Europa de la alta velocidad ferroviaria (en trazo más grueso la línea de alta velocidad y en trazo más fino la línea convencional utilizada por el Tren de Alta Velocidad). Fuente: S.N.C.F.

La densa red urbana de las zonas servidas por el TAV en Italia y Alemania no permite privilegiar la velocidad frente a la frecuencia. En efecto, se ha optado por que el mayor número posible de ciudades posea un servicio de calidad y se ha hecho un hincapiél en la búsqueda de poderosas aceleraciones y deceleraciones, más que en el dominio de la velocidad. Por el momento, la alta velocidad ferroviaria de estos países no busca la creación de una red ferroviaria a parte. Al contrario, existe la voluntad de fomentar el aumento de velocidad en el conjunto de la red ferroviaria mediante Planes de Velocidad (Schnellfahrstreckenplan o el Plan Integrativo italiano). Todo esto se ha conseguido gracias a la dotación de las nuevas líneas de al ta 
velocidad de un perfil muy suave, con una pendiente máxima de solo 12â, lo que permite que el conjunto de los trenes convencionales de viajeros o de mercancías puedan circular por la nueva vía. En efecto, existe una compatibilidad total entre las líneas nuevas y las líneas clásicas, lo que lleva consigo un fuerte coste adicional. Esta compatibilidad está reforzada por un trazado paralelo a la vía clásica, así como por múltiples obras de conexión entre las dos redes (AUPHAN, 1992 y ZEMBRI, 1993).

\section{Los sistemas francés y español}

Estos sistemas se caracterizan por una voluntad menos firme de dotar al conjunto de la red de un aumento de la velocidad. En efecto, la conectividad es en este caso unilateral. Bien es el TAV el que penetra en la red clásica, como es el caso del TGV francés, o bien es el tren tradicional, el Talgo 200, el que utiliza la línea de alta velocidad. Esta menor integración entre las redes es fruto de algunos factores, como por ejemplo la existencia de densidades de población y de urbanización relativamente bajas y la presencia de una red urbana menos equilibrada que en el caso anterior.

En la concepción francesa y, en menor medida, en la española, el problema de dotar a una localidad de un servicio de alta velocidad, se remite a una estimación del balance financiero. Este balance financiero pone en comparación el coste comercial, esto es, la pérdida eventual de clientela que engendra el aumento de la duración de trayecto resultante del tiempo consumido por la parada, y la ganancia debida al aumento de la población a servir. De esta manera, las posibilidades de que una aglomeración urbana obtenga una parada son directamente proporcionales a la masa de población e inversamente proporcionales al tiempo de trayecto consumido para servirla (AUPHAN, 1992 ). En efecto, la clientela del tren de alta velocidad es cada vez más reticente a utilizar el TAV si aumentan las paradas intermedias entre su lugar de origen y su destino, es decir, si existe un aumento de tiempo consumido en su viaje.

Con este estado de las cosas, se comprende el interés que tienen los sistemas de alta velocidad francés y español para aprovechar al máximo la travesía de la tierra de nadie sin hacer ninguna parada, para dotar de servicio solamente a las ciudades más importantes. Sin embargo, la presión de la opinión pública y una voluntad por favorecer una mayor justicia socioterritorial, han conseguido matizar este concepto economicista. De esta manera, pequeñas ciudades como Puertollano y Ciudad Real poseen un servicio de calidad gracias a los AVE lanzadera.

Por su parte, la S.N.C.F. (Ferrocarriles Franceses) ha instalado estaciones en lugares estratégicos, bien en mitad del campo o bien en pequeñas ciudades que puedan hacer la función de punto de servicio para una población dispersa. Las iniciativas llevadas a cabo para crear polos de desarrollo alrededor de estas estaciones bis y 
regionales, han conocido un fracaso, generalmente debido a un escaso realismo de los proyectos y al carácter fuertemente condicionante que tienen el contexto donde se inscriben, demasiado periférico.

Por otra parte, como ya se ha señalado, en el sistema francés, a diferencia del italiano y del alemán, existe una conexión unilateral entre las dos redes ferroviarias. Esto es, la nueva maquinaria ferroviaria puede recorrer las líneas nuevas y las líneas clásicas pero el tren tradicional no puede utilizar la nueva infraestructura como consecuencia de las elevadas pendientes de la línea. La existencia de estas fuertes pendientes se justifica por un sustancial ahorro de dinero en la construcción de la línea, al evitarse la construcción de numerosas obras de fábrica. En el caso español, no es el valor de la pendiente, similar a la de los sistemas alemán e italiano, sino la existencia de anchos de vía diferentes lo que impide al material rodante de alta velocidad ir más allá de Madrid o Sevilla.

\section{EL IMPACTO URBANO DE LA ALTA VELOCIDAD EN ESPAÑA Y FRANCIA}

Cuando se habla de impacto, no hay que pensar en un acontecimiento brusco que viene a irrumpir radicalmente en la situación previa. Por el contrario, este impacto urbano se encuentra estrechamente imbricado en el sistema en el cual se instala.

De esta manera, podemos distinguir dos tipos de impactos: aquellos que se manifiestan de una manera clara en el territorio, en un plazo de tiempo relativamente corto, y aquellos que tan sólo pueden ser detectados mediante un análisis más profundo y que solo comienzan a manifestarse con claridad después de 10 o 15 años de la puesta en servicio de una línea de alta velocidad. De esta manera, únicamente la línea TGVSudeste nos proporciona en la actualidad datos clave a este respecto.

\section{Los impactos directos}

Son aquellos que se manifiestan de manera más inmediata y que se presentan de una manera más clara. Los impactos directos más importantes son los cambios de la accesibilidad y la remodelación del barrio de la estación.

1.- Una nueva accesibilidad y un nuevo competidor en el mercado de los transportes

Según un estudio realizado en Francia en 1993, el TGV sólamente modifica de manera sustancial la accesibilidad de las ciudades que poseen un servicio en el cual lo esencial del recorrido se realiza por línea de alta velocidad o línea clásica 
acondicionada. La accesibilidad de la red ferroviaria francesa ha conocido una mejora general de la accesibilidad que ha servido de provecho esencialmente a la capital. En efecto, la brecha que separa los altos valores de accesibilidad de París de los valores de casi la totalidad de las ciudades francesas se ha incrementado notablemente (AUPHAN et al., 1993).

Las ciudades que no tienen servicio directo registran una tendencia más o menos acusada a ver su servicio ferroviario degradado. Este fenómeno explica el enorme interés de las administraciones locales por obtener un punto de servicio sobre la nueva línea.

Conviene señalar que el aumento de la accesibilidad de una localidad gracias a la alta velocidad no tiene por que estar forzosamente ligada a que el TAV de servicio directamente a su núcleo urbano. En realidad, lo que ha de ser tomado en cuenta es la calidad del servicio en términos de duración del recorrido, de frecuencias, la riqueza de destinos, etc. Dentro de este debate, existe una línea de opinión que tiende a subrayar la importancia de la mejora de las relaciones interregionales. En efecto, la prioridad de las ciudades que no tienen opción de ser servidas directamente, debe ser la mejora de sus transportes ferroviarios regionales para conectar de forma cómoda con las localidades que tienen el tren de alta velocidad (Plassard citado en EMMANGARD, 1990).

La accesibilidad por tren tradicional y por avión se degrada mury a menudo de forma considerable. Por ejemplo, antes del AVE, las partes de mercado de la conexión Madrid - Sevilla estaban repartidas de la siguiente manera: automóvil $60 \%$, autocar $15 \%$, ferrocarril $14 \%$ y avión $11 \%$. Desde 1992, estos porcentajes han sido redistribuidos como sigue: AVE $48 \%$, automóvil $37 \%$, autocar $9 \%$, avión $4 \%$ y tren convencional 3\% (POINGT et al., 1996).

Por otra parte, la puesta en marcha de la alta velocidad es susceptible de degradar ciertas conexiones ferroviarias. Por ejemplo, los trenes de largo recorrido París - Lyón - Midi, que utilizaban la línea clásica, han sido reemplazados por los TGV directos entre la capital y las ciudades meridionales, produciendo una reducción del número de trenes que conectan Lyon a Avignon y a Marsella. Este trasvase de tráfico sobre la nueva línea ha provocado igualmente una disminución de los trenes que aseguran el servicio de Dijon y de Ginebra.

Por otra parte, el TAV crea un tráfico inducido y modifica las prácticas de desplazamiento. Entre París y Lyon, la parte correspondiente a los viajes de ida y vuelta realizados en el día ha pasado de $25 \%$ en 1980 a $33 \%$ en 1990 y la de los desplazamientos durante la media jornada, de 1 a $4,4 \%$, permitiendo aumentar la frecuencia de los desplazamientos de los efectivos de las empresas terciarias en un $59 \%$ 
Todo ello ha sido posible gracias a la cadencia del servicio que confiere al TAV una mayor flexibilidad de utilización que el avión.

\section{2.- La remodelación del barrio de la estación}

Algunas ciudades han aprovechado para crear ex novo un nuevo barrio o un nuevo polo central, como muestra el caso de Lille. Entre estos dos tipos de operaciones, otras aglomeraciones han optado por explotar la llegada del TAV para reestructurar o remodelar su estación y entorno urbano (Ciudad Real, Le Mans o Poitiers, por ejemplo).

El análisis de los diferentes proyectos o realizaciones permite establecer una caracterización según tres constantes:

- Las estaciones situadas a proximidad inmediata del hipercentro conocen un efecto real de redinamización que tiene por corolario el refuerzo de los sistemas de acceso al centro (transportes urbanos, suburbanos y regionales). Algunas de estas ciudades han acompañado la integración urbana de la alta velocidad con un proyecto de reestructuración de sus transportes públicos.

- El haz ferroviario heredado del siglo XIX por la mayor parte de las aglomeraciones urbanas, representa un corte en el tejido urbano. El efecto del tren de alta velocidad lleva consigo un notable crecimiento de los precios del sector inmobiliario, pero permite dotarse de nuevas comunicaciones y, en ciertos lugares, 1 a cobertura de las vías, como es el caso de Lille. Esto permite unir los barrios que hasta el momento funcionaban sin una complementariedad real.

- La emergencia de nuevos útiles que ponen en conjunto los diferentes estudios y operaciones, permiten reunir y hacer trabajar juntos a todo el conjunto de los múltiples actores que están directamente relacionados en la elaboración de los proyectos (ayuntamientos, sociedades de ferrocarril, promotores, etc.) (ADUAN, 1994).

El contexto donde se llevan a cabo estas operaciones condiciona en gran medida su éxito. Las medidas llevadas a cabo en los alrededores de las estaciones de Vendôme, Mâcon o Montchanin, situadas en zonas rurales, pueden ser consideradas como un fracaso. Sin embargo, la remodelación del barrio de la estación TGV de Lyon, la PartDieu, ha permitido la creación de un nuevo hipercentro que ha liberado al casco histórico de un elevado número de las funciones que lo saturaban, produciéndose, de hecho, un reparto funcional entre los dos polos centrales. La operación espectacular de Lille y el barrio de la estación, Euralille, es de sobra conocida. En este caso se ha aprovechado la llegada de la alta velocidad para redinamizar, con éxito, el barrio de la estación en muchos otros casos. 
La falta de espectacularidad de ciertas operaciones llevadas a cabo cuando la alta velocidad da servicio a la zona central de las aglomeraciones urbanas, suele ser explicable por razones coyunturales. Los dos mejores ejemplos de esta categoría de ciudades los ofrecen Tours y Sevilla. Tours centró la remodelación del barrio de la estación en el Palacio de Congresos y no en el nuevo servicio debido a que la estación principal de la aglomeración se encuentra a algunos Kilómetros del centro, en SaintPierre-des-Corps y no en el mismo Tours. En el caso de Sevilla, las estrategias urbanas principales no podían centrarse en el barrio de la estación debido a que en ese mismo momento, la mayoría de las iniciativas se volcaban en la Exposición Universal de 1992.

Sin duda estas operaciones urbanas constituyen el efecto más claramente visible del TAV, que sirve como pretexto para revitalizar un barrio generalmente degradado que suele aprovechar la imagen de modernidad que proporciona el nuevo tren.

\section{Los impactos indirectos}

Los impactos indrectos hacen referencia al papel que juega el TAV para atraer implantaciones de empresas de terciario superior y sobre el turismo. Son los más difícilmente identificables por varias razones. Por un lado, necesitan cierto tiempo para mostrarse y además de esto solo pueden ser identificables mediante un trabajo de investigación. Otro de los problemas reside en la posibilidad de desagregar el verdadero impacto que resulta de la alta velocidad del de otros factores, teniendo en cuenta que interviene en gran medida la subjetividad del investigador y de la entidad entrevistada.

1.- El impacto sobre la localización de empresas del sector terciario superior

A este respecto, ha sido realizado un importante estudio sobre las ciudades de la línea TGV-Sudeste, basado en los resultados que se recogen de las encuestas realizadas a las empresas de terciario superior instaladas en las ciudades y en el barrio de la estación tras el establecimiento del servicio de la alta velocidad. Los resultados han de ser tomados con cautela debido a la escasa representatividad del número de respuestas dadas a los cuestionarios por parte de las empresas (MANNONE, 1995).

Los resultados de la investigación muestran el reducido papel de la alta velocidad como localizadora de actividades. Parece ser que el TGV no ha resultado ser una máquina centralizadora como temían los pesimistas, ni un elemento de reparto de las actividades, como creían los optimistas. En efecto, el tren de alta velocidad no suele considerarse nunca como el principal motivo de localización por parte de las empresas encuestadas. 
La influencia del TGV está muy ligada a la calidad de la oferta ferroviaria y solo ha constituido un factor de localización para las ciudades que se han beneficiado de un notable acercamiento a París en lo que concierne a las frecuencias y al tiempo de viaje.

Por otra parte, las empresas utilizan la alta velocidad sobre todo como un instrumento al servicio de su estrategia, es decir, solo lo consideran como un factor de localización de importancia si su mercado es nacional o europeo. Cuando el área de mercado de estas empresas es regional, la alta velocidad deja de constituir, obviamente, un factor de atracción, teniendo en cuenta que este modo de transporte no ha sido concebido para las relaciones a corta distancia.

El ejemplo más interesante lo constituye el análisis de Lyon y el barrio de su estación, la Part-Dieu. La doctoranda Valérie Mannone realizó a mediados de los años noventa una serie de encuestas que dieron como resultado la elaboración de la siguiente tipología:

- Empresas instaladas en la Part-Dieu a causa del TGV. Más de la mitad de entre ellas son antenas parisinas instaladas en la Part-Dieu entre 1985 y 1990. Su mercado es local y regional y se extiende, para la mitad de entre ellas, a todo el cuadrante sudeste.

Si bien el TGV ha sido un factor determinante, solo actúa como único criterio de elección para un reducido número de empresas. La mitad de ellas utilizan la alta velocidad más de una vez por semana para realizar encuentros en el seno de la sede parisina o visitas a su clientela meridional. Las otras solo van a París una vez o dos por mes. Estas empresas no han conocido una extensión de su área de mercado: son antenas de empresas parisinas que han sacado provecho del servicio TGV para implantarse en el mercado lionés y extender así su área de influencia a todo el sudeste.

- Empresas para las que el TGV ha sido un factor de localización secundario. Casi la mitad de ellas pertenecen a firmas con varios establecimientos cuya sede es parisina. Se trata, para la mayor parte, de antenas de grandes gabinetes jurídicos, de seguros y asesorías, cuya área de influencia va solo excepcionalmente más allá del marco regional.

Su implantación en la Part-Dieu es la más antigua. La elección del barrio de la estación es el resultado de la atractividad del centro de negocios que ocupa una posición central en el corazón de una región que presenta un tejido económico diversificado, y para empresas que provienen del centro tradicional, de la necesidad de ampliarse en un lugar de fácil acceso. 


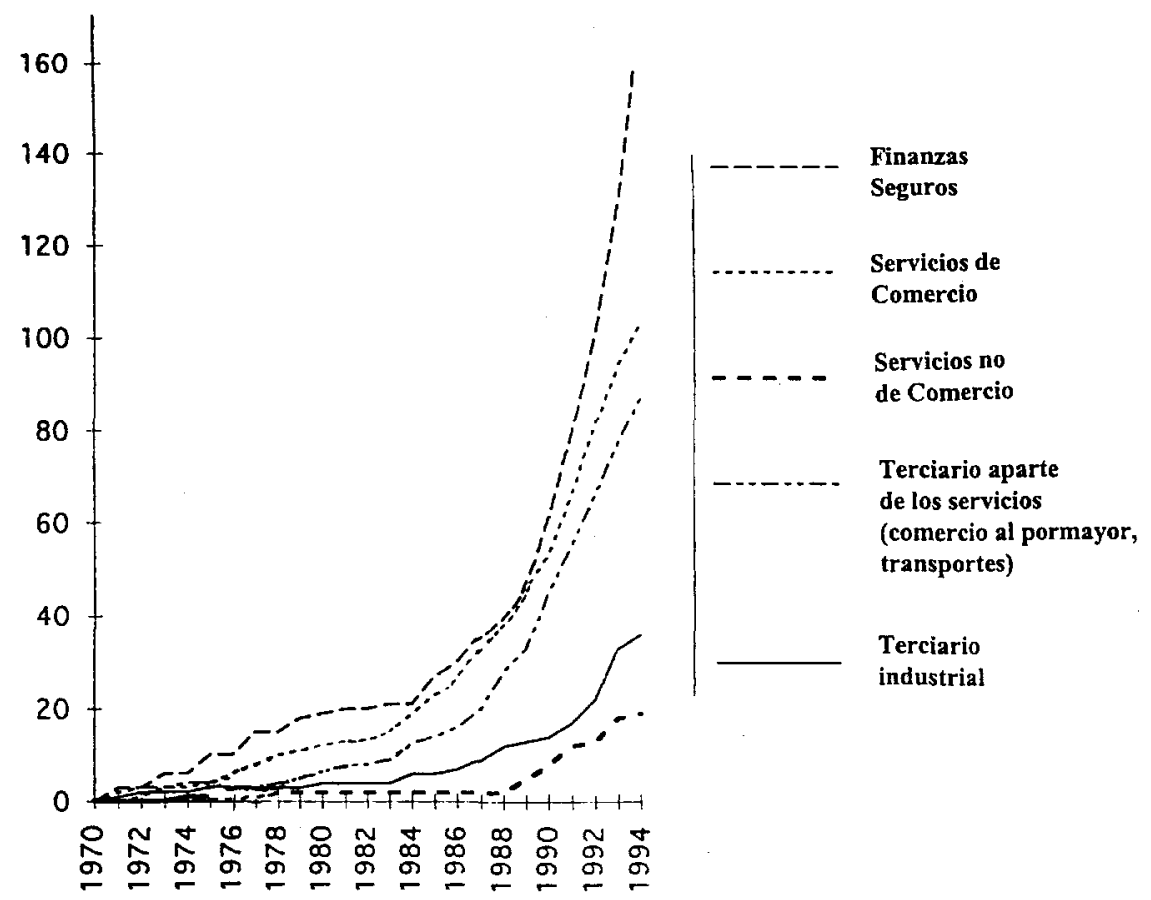

Figura 2.- Evolución de la ocupación del centro direccional de la Part-Dieu (1971-74). Fuente: MANNONE (1995), según el fichero de empresas de la C.C.I.

La alta velocidad ha sido igualmente tenida en cuenta en la creación de establecimientos cuya sede se encuentra en otras regiones o en el extranjero. Se trata de empresas de transporte o de comercio al por mayor cuyo mercado es nacional o internacional. Estos establecimientos se han implantado recientemente con el objetivo de conquistar el mercado regional y han elegido la Part-Dieu en razón de su situación central y de la cualidad de la oferta de las oficinas.

- Empresas en las que el TGV ha jugado un papel desdeñable. Estas empresas han tomad en consideración, principalmente, el mercado, el barrio de negocios y el tejido económico e industrial de la región. La mayor parte de las empresas de esta tercera categoría son sociedades lionesas cuyo mercado es esencialmente ronalpino, y $30 \%$ solamente tienen un mercado que va más allá del marco regional, (MANNONE, 1995).

\section{2.- La alta velocidad ferroviaria y el turismo}

Puede afirmarse que la mejora general de los medios de transporte favorece el desarrollo de los flujos turísticos. Sin embargo, la pobreza de los datos estadísticos y la dificultad para discernir la influencia del TAV de la de otros modos de transporte, 
hace muy difícil determinar el verdadero impacto de la alta velocidad en el turismo de las ciudades que poseen este servicio.

La alta velocidad favorece la aparición de nuevos tipos de desplazamiento turístico. En las relaciones que se han beneficiado de una fuerte reducción del tiempo de viaje y de un servicio de calidad, la posibilidad de efectuar el viaje de ida y vuelta durante el día, provoca una disminución del número de pernoctas. En la mayor parte de las ciudades que poseen servicio de alta velocidad, la duración de las estancias hoteleras se ha reducido. Sin embargo, el TAV no es el único responsable de las dificultades que atraviesa la hostelería de los centros de las ciudades, sobre todo francesas. La crisis económica y la multiplicación, junto a las encrucjjadas de las autopistas, de los hoteles baratos, han tenido una influencia mucho más determinante en esta evolución.

Por otra parte, la puesta en marcha del francés TGV se ha acompañado de un desarrollo de nuevos productos turísticos que incluyen el transporte de los clientes por alta velocidad, pero solo han conocido un corto éxito debido a la banalización que $h$ a experimentado la nueva oferta de transporte y al cambio de la política de las agencias de viaje en favor de las compañias de autocares.

Independientemente de estas operaciones promocionales, el TAV favorece e1 turismo de negocios y la actividad de congresos en particular. La mayor parte de las ciudades servidas se han apoyado en la nueva oferta ferroviaria para desarrollar la actividad de congresos. El papel de la alta velocidad en esta evolución ha de ser evaluado en su justa medida. El renombre de las ciudades, la calidad de sus infraestructuras de acogida y su accesibilidad general, así como una promoción agresiva, continúan siendo los principales factores que condicionan el éxito de estas actividades.

En resumen, el tren de alta velocidad solo tiene un impacto secundario en el desarrollo del turismo de negocios y de otras formas de ocio en general. Su influencia continúa siendo dependiente de las potencialidades locales (MANNONE, 1995).

\section{CONCLUSIÓN}

Con esta perspectiva, la ciudad de Zaragoza va a ser próximamente servida por la alta velocidad. Según los estudios más recientes, puede afirmarse que existen cuatro factores clave que definen las potencialidades de la demanda sobre un lugar servido por el TAV. Dentro del ámbito regional, el análisis de la competencia de las otras estaciones de la línea, (por ejemplo Lerida), es un elemento determinante para 
orientar el proyecto urbano. Se trata de estudiar la calidad de la competencia (número y precio del metro cuadrado de las oficinas que han sido proyectadas en las otras ciudades de la línea), para aprovechar el efecto de la alta velocidad y saber que complementariedad buscar.

De igual manera, es importante que la región y, en general, todo el corredor del Ebro puedan aprovechar el efecto alta velocidad. Esto solo se logrará si existe una buena coordinación entre los servicios de autobús interurbano y tren convencional con el TAV. Es aquí donde la estación intermodal juega un papel excepcional para hacer que la alta velocidad suponga para las zonas rurales algo más que una infraestructura parasitaria que además degrada el servcio por tren convencional. Con un servicio intermodal de calidad, no solo salen ganando aquellas localidades no servidas directamente por el TAV. El incremento de cientela donde el tren hace parada hace más interesante para la compañía explotadora aumentar el número de paradas en la estación que recibe la alta velocidad.

Otro factor clave es la calidad del servicio, que tendrá un efecto directo sobre la demanda inmobiliaria de las empresas. Por debajo de cierto umbral de frecuencias, puede afirmarse que el interés de una implantación en el barrio de la estación se convertirá en más que dudoso.

La calidad de la arquitectura del proyecto estación y su inscripción en el paisaje urbano es un elemento determinante en la capacidad para atraer a los inversores. Pero no hay que olvidar que esta calidad es solo un elemento más. El soporte sobre el cual debe reposar toda estación de alta velocidad ha de estar imperativamente constituido por la calidad de la intermodalidad con el conjunto de las redes de desplazamiento. Esta intermodalidad ha de ser amplia, fácilmente legible y de calidad.

El cuarto factor clave es la demanda regional. Las primeras y, a menudo, la mayor parte de las implantaciones en el barrio de la estación, provienen de traslados de empresas que se encuentran enclavadas en el tejido urbano, con una oferta de débil calidad y que desean relocalizarse en el marco de un proyecto estructurado y que goza de una buena imagen. El análisis de una oferta insatisfecha es por lo tanto un dato de primera calidad para abordar el estudio. Pero es necesario también ofrecer en las operaciones realizadas en el barrio de la estación servicios suplementarios que no se encuentran en otros lugares.

La ciudad de Zaragoza cuenta con la experiencia que otorga el resultado del impacto de la alta velocidad en otras ciudades, comenzamos a conocer mejor los blancos hacia los que hay que orientar los proyecto de desarrollo para sacar el mejor provecho del servicio del TAV. 


\section{BIBLIOGRAFÍA}

ADUAN (1994): Gare TGV. Le produit immobilier. Documento interno, inédito.

AUPHAN, E. (1992): "La grande vitesse ferroviarie de part et d'autre du Rhin: face à face ou rapprochement?", Révue géographique de l’Est, 4, 257-272.

AUPHAN, E. et al. (1993): "De la distance-temps à la distance-coût." En BONNAFOUS, A., Circuler Demain, 143-156.

BONNAFOUS, A. (1981): "Les effets économiques indirects du TGV", Le courrier du C.N.R.S., 1981, $42,25-33$.

EMANGARD, P. H.. (1990): "Destination Bordeaux", La vie du Rail, 2261, 8-10.

MANNONE, V. (1995): L'impact régional du TGV Sud-Est. Tesis doctoral inédita.

POINGT, M. H., et al. (1996): "Le train face à l'avion", La vie du rail, 2545, 16-25.

ZEMBRI, P. (1993): "TGV-Résseau ferré classique: des rendez-vous manqués?", Annales de Géographie, 571, 282-295. 